\title{
Managing female urinary incontinence: A regional prospective analysis of cost-utility ratios (curs) and effectiveness
}

\author{
Elisabetta Costantini ${ }^{1}$, Massimo Lazzeri ${ }^{1}$, Vittorio Bini ${ }^{2}$, Alessandro Zucchi ${ }^{1}$, Emanuele Scarponi ${ }^{1}$, \\ Massimo Porena ${ }^{1}$ \\ ${ }^{1}$ Department of Medical-Surgical Specialties and Public Health, Urology and Andrology Section, University of Perugia, Perugia, Italy; \\ ${ }^{2}$ Department of Internal Medicine, University of Perugia, Perugia, Italy.
}

\begin{abstract}
Summary Introduction: To evaluate the cost-utility of incontinence treatments, particularly anticholinergic therapy, by examining costs and quality-adjusted life years.

Materials and methods: A prospective cohort study of women who were consecutively referred by general practitioners (GPs) to the Urology Department because of urinary incontinence. The primary outcome was evaluation of the cost-utility of incontinence treatments (surgery, medical therapy and physiotherapy) for stress and/or urgency incontinence by examining costs and quality-adjusted life years.

Results: 137 consecutive female patients (mean age 60.6 \pm 11.6; range 36-81) were enrolled and stratified according to pathologies: SUI and UUI. Group A: SUI grade II-III: 43 patients who underwent mid-urethral sling (MUS); Group B: SUI grade I-II 57 patients who underwent pelvic floor muscle exercise and Group C: UUI: 37 patients who underwent antimuscarinic treatment with $5 \mathrm{mg}$ solifenacin daily. The cost utility ratio (CUR) was estimated as saving more than $€ 1200$ per QALY for surgery and physiotherapy and as costing under $€ 100$ per $Q A L Y$ for drug therapy.

Conclusions: This study shows that appropriate diagnosis and treatment of a patient with incontinence lowers National Health Service costs and improves the benefits of treatment and quality of life.
\end{abstract}

KEY WORDS: Female urinary incontinence; Cost-utility analysis; Anti-muscarinic therapy; Mid urethral slings; Physiotherapy, QALY.

Submitted 30 September 2014; Accepted 31 December 2014

\section{INTRODUCTION}

Female urinary incontinence (UI) affects between 10\% and $60 \%$ of women worldwide (1-2). As a major health problem it has a marked social impact, with significant worsening of quality of life (QoL) and high costs for national health system (NHS), which vary with the country (3). Incontinence imposes very different expenses on payers, providers and patients which can lead to complex arrangements for where patients get care and what services are covered by health insurance, thus ultimately determining outcomes for patients (4). As the average life-span of Western populations is rapidly lengthening, the prevalence of incontinence will continue to rise and the demand for incontinence services will increase (5). Since costs are assuming a greater role in health-care decision-making, the aim of the present study was to evaluate the cost-utility of three incontinence treatments by examining costs and quality-adjusted life years. Since the type of therapy varies with the nature of incontinence, our objective in this prospective cohort study was not to compare different strategies of incontinence therapy but to determine whether each therapy is cost-useful. This type of assessment is of particular interest in Italy as the Italian NHS does not fund anticholinergic drugs.

\section{MATERIAL AND METHODS}

Study design

A prospective descriptive observational study with no inter-group comparisons was designed and approved as a "Research Project" by the "Umbria Region" (ANNEX) to test an innovative organizational model of UI treatment in an attempt to reduce costs and increase patient satisfaction.

The approval to conduct the study (number 718) was obtained by Regional research scientific committee with the protocol number 0096568, classification XVII.4.

Before starting the study general practitioners (GPs) in the No 2 Local Health Board in Perugia (Italy) were asked how many patients used NHS-supplied pads. Approximately $8 \%$ used them, but only about $2 \%$ were suitable for recruitment to the present study as the others were very elderly or had other major pathologies such as double incontinence or were bedridden.

The study was conducted on women who were consecutively referred by GPs to the Urology and Andrology Division (University of Perugia) because of UI. Patients were affected by stress urinary incontinence (SUI), urge urinary incontinence (UUI), mixed urinary incontinence

No conflict of interest declared.

Archivio Italiano di Urologia e Andrologia 2014; 86, 2 
(MUI). In patients with mixed incontinence, the dominant symptom of either stress or urge incontinence was treated. Patients were divided into three groups according to the type and severity of incontinence, in accordance with International Continence Society (ICS) criteria and the Ingelman Sunderberg classification (6) and were treated with surgery, drugs or physiotherapy. Only patients who gave written informed consent to participating in this observational study were included in each group. All information was obtained using validated questionnaires: the IIQ 7 and UDI6 questionnaires for outcome evaluation and Life expectancy EQ-5D (see quality of life below) for quality of life evaluation at baseline and at the 3 month follow-up. Pad use pre- and post-treatment was also included in the analysis

The primary outcome was evaluation of the cost-utility of 3 incontinence treatments by examining costs and quality-adjusted life years without an inter-group statistical comparison. Our aim was to assess the cost-utility of each strategy and its impact on the Italian National Health Service bearing in mind that each treatment was not funded in the same way.

\section{Cost Utility Analysis (CUA)}

Cost is typically measured in currency, and should reflect the present value of total future expenditures that would be incurred by making a certain decision. Utilities are typically measured in Quality Adjusted Life Years (QALYs). A QALY accounts for the morbidity of a health state, based on the assumption that a year of life with morbidity is not equal to a year of life without morbidity. The value of a year with morbidity, the "utility weight", can be determined through validated survey instruments.

To calculate the cost-utility ratios for incontinence therapy we calculated quality of life and costs. Cost-utility ratios are normalized values that reveal how much money (cost) must be paid for a single extra QALY (utility). When deciding between two management options, one should consider the cost of the gain of QALYs. This ratio, the cost-utility ratio, is calculated as (Cost 1 Cost2)/(QALY1-QALY2). If one of the interventions costs less and yields more QALYs, that decision is "cost-saving" and "prevails over" the other option. Confidence intervals for the cost per QALY ratio were estimated using the non-parametric bias-corrected percentile bootstrap method (7). Because of the limited degree of modeling in this cost utility analysis, we carried out sensitivity analyses only on the use of different utility measures: EQ-5D index and EQ-5D visual analogue scale (VAS).

\section{Quality of life}

Health-related QoL was assessed by patients before therapy and 3 months after treatment by means of the Life expectancy EQ-5D questionnaire, a non-disease-specific self-report instrument for measuring health-related QoL. It consists of the EQ-5D self-classifier and the EQ-5D visual analogue scale ranging from 0 to 100 (8). Patients classify their own health status in five dimensions: mobility, selfcare, usual activities, pain/discomfort, and anxiety/depression. Answers are given on a three point scale: $1=$ no problems, 2 = moderate problems, and $3=$ severe problems. Theoretically, 243 health statuses could be generated by this classification. Each health status can be given a value from -0.59 to 1.0 by means of the time-trade method developed for UK population (9). As incontinence does not impact on life expectancy, QALY was calculated for each patient by subtracting her age at the start of the study from the age-specific life-expectancy values relative to the general female population in the Umbria region in 2010 (source ISTAT, National Institute of Statistics) and then adjusted for EQ-5D scores. No further or additional adjustment was done. Two types of QALY was obtained as one is calculated on the basis of the descriptive self-classifier and the other on the VAS scores.

\section{Costs}

According to our regional legislation urodynamic tests are performed only in patients who are candidates for surgery and all the other tests and procedures are performed under an accurate spending review in order to contain costs as much as possible.

Direct costs, based on prices from the Italian Diagnosis Related Groups (DRG), were calculated for:

\section{Diagnosis}

Out-patient appointments and laboratory tests

Urodynamic testing (only in the surgery group)

2. Treatment and/or prevention

Drug therapy (recommended pharmaceutical company prices)

Hospital stays in the ward and in the day hospital

Surgical intervention

Physiotherapy

3. The following costs could not be quantified in our study

Treatment for incontinence-related skin infections

Treatment for urinary tract infections

These costs were derived from the Arlandis-Guzman study published on BioMed Central Ltd. in 2011 (10).

All costs are presented as mean costs evaluated in 2011. As the Italian National Health Service does not cover the costs of anticholinergic therapy the Astellas company kindly provided the 3 months supply to avoid patients stopping therapy because of the cost of the drugs.

We expected to find that no single treatment was worse than any other (the null hypothesis) or that all treatments, though not to the same measure, reduced pad use and incontinence-related complications, thus lowering costs and providing a gain in QALY.

\section{Statistical analysis}

Pre- and post- incontinence treatment data from the three intervention groups were analyzed using the Wilcoxon non-parametric tests for paired discrete data. Since the type of therapy varies with the nature of incontinence, our objective in this prospective cohort study was not to compare incontinence treatments but to determine whether each was cost-useful. All analyses were performed using IBM SPSS release 20.0.0, 2011, with significance level set at $\mathrm{p} \leq 0.05$.

\section{ResULtS}

From January 2010 to December 2011 GPs identified 190 patients as suitable for recruitment to this study. 
Table 1.

Flow chart of patients' distribution across the three different categories

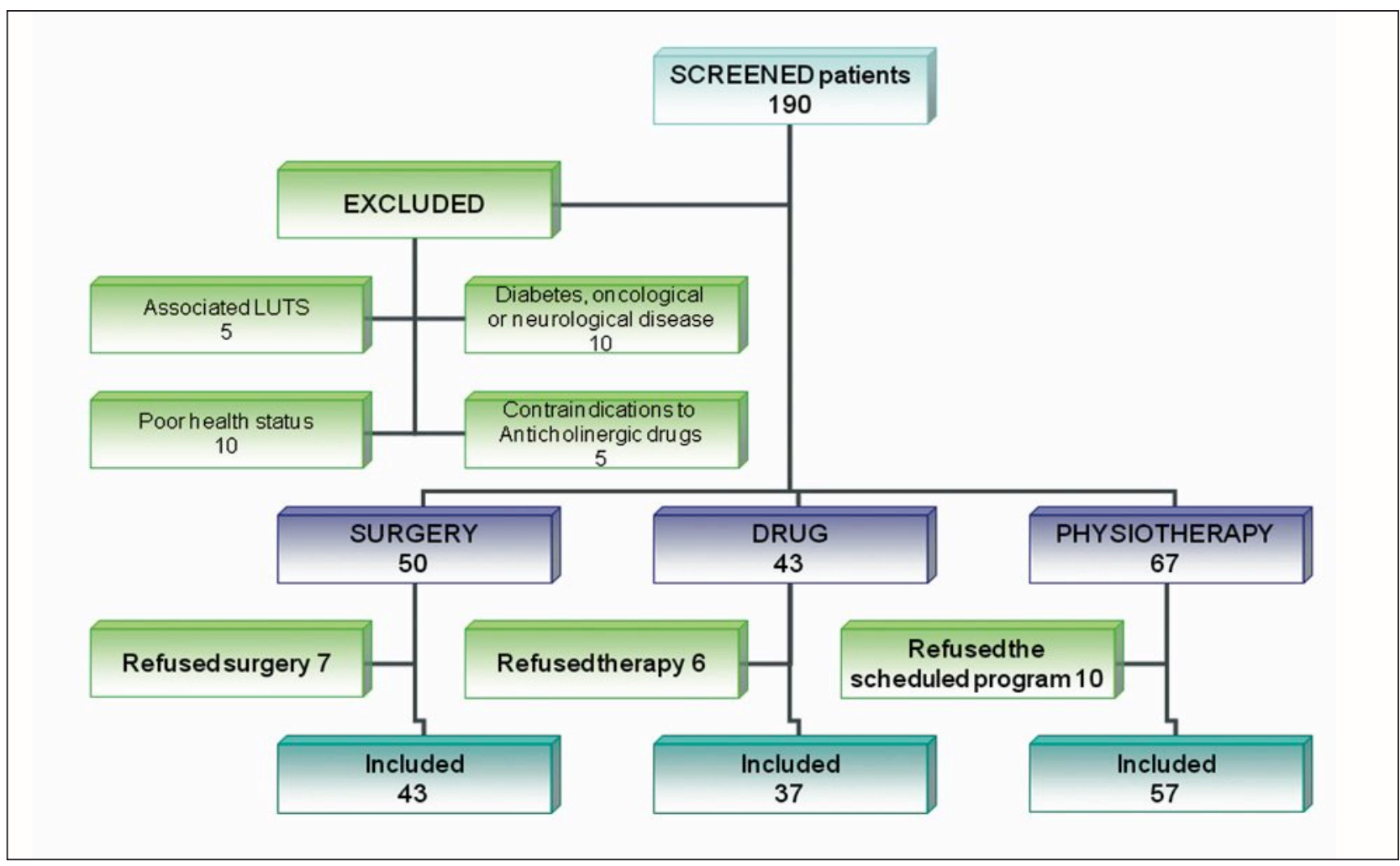

After a complete urogynaecological examination the senior urologist divided the patients into three groups according to the type and severity of incontinence. Pelvic floor exercises were recommended for patients with mild SUI, mid-urethral sling surgery for patients with moderate-severe SUI, and anticholinergic therapy for patients with UUI or MUI with urgency as predominant symptom. Figure 1 illustrates the enrolment process and the patients who were included in the study.

Thirty of the 190 patients were excluded: 10 because poor health status prevented routine check-ups or patients were unable to understand or respond to the questionnaires: 10 because of diabetes, oncological or neurological diseases; 5 in the anticholinergic drug therapy group because of contraindications and 5 because of associated low urinary tract symptoms (LUTS). Of the remaining 160 patients, 50 were assigned to the surgery group, 67 to the physiotherapy group and 43 to the drug group.

Ultimately only 137/160 female patients (mean age 60.6 \pm 11.6; range 36-81) gave written informed consent and were enrolled according to the UI type and severity. Group A: SUI grade II-III: 43 patients (mean age $61 \pm 12$; range 38-80 years) underwent mid-urethral sling (MUS); 7/50 refused surgery. Group B: SUI grade I-II 57 patients (mean age $66 \pm 10$; range 49-79 years) undertook a pelvic floor muscle exercise programme; 10/67 refused to participate and perform the exercises regularly for at least 3 months. Group C: UUI: 37 patients (mean age 58 \pm 11 ; range 36-77 years) who underwent antimuscarinic treatment with solifenacin $5 \mathrm{mg}$; 6/43 refused drug therapy ( 3 because of constipation risk, and 3 because of undeclared personal reasons). No patients were lost during the 3 month follow-up, The principal reasons for not having any drop-outs were the relatively short follow-up and free supply of medication in the drug group. In the surgery and physiotherapy groups additional check-ups at 6 and 12 months confirmed the 3-month results.

Table 1 shows the estimated costs for each group. Table 2 shows questionnaire scores and pad use before and after each treatment. Scores improved significantly after treatment in all 3 groups. Improvements were confirmed by the cost utility ratio (CUR) assessment according to both sections of the EQ-5D questionnaire (Table 3). The cost was then modified to include pad use (Table 4) and the cost of treating skin and urinary tract infections before and after treatment (Table 5).

Table 1.

Estimated costs applied for each group.

\begin{tabular}{|lccc|}
\hline Estimated costs $(\boldsymbol{€})$ & Surgery & Drug & Physiotherapy \\
\hline Out-patient appointments ${ }^{\mathrm{a}}$ & 33.06 & 33.06 & 33.06 \\
Urodynamic testing & 56.81 & & \\
Uroflussimetry & 11.62 & 11.62 & 11.62 \\
$\begin{array}{l}\text { Drug therapyb } \\
\text { Surgical intervention }\end{array}$ & 4324.00 & & \\
$\begin{array}{l}\text { Physiotherapy } \\
\text { Pade }\end{array}$ & 0.31 .90 & 0.31 & 0.31 \\
\hline $\begin{array}{l}\text { a: } 2 \text { appointments; b: recommended pharmaceutical company prices for } 1 \text { months } \\
\text { of therapy; c: included hospital stay; d: 24 sessions; e: included waste disposal. }\end{array}$ \\
\hline
\end{tabular}


Table 2.

Questionnaire scores and the pad use before and after each type of therapy.

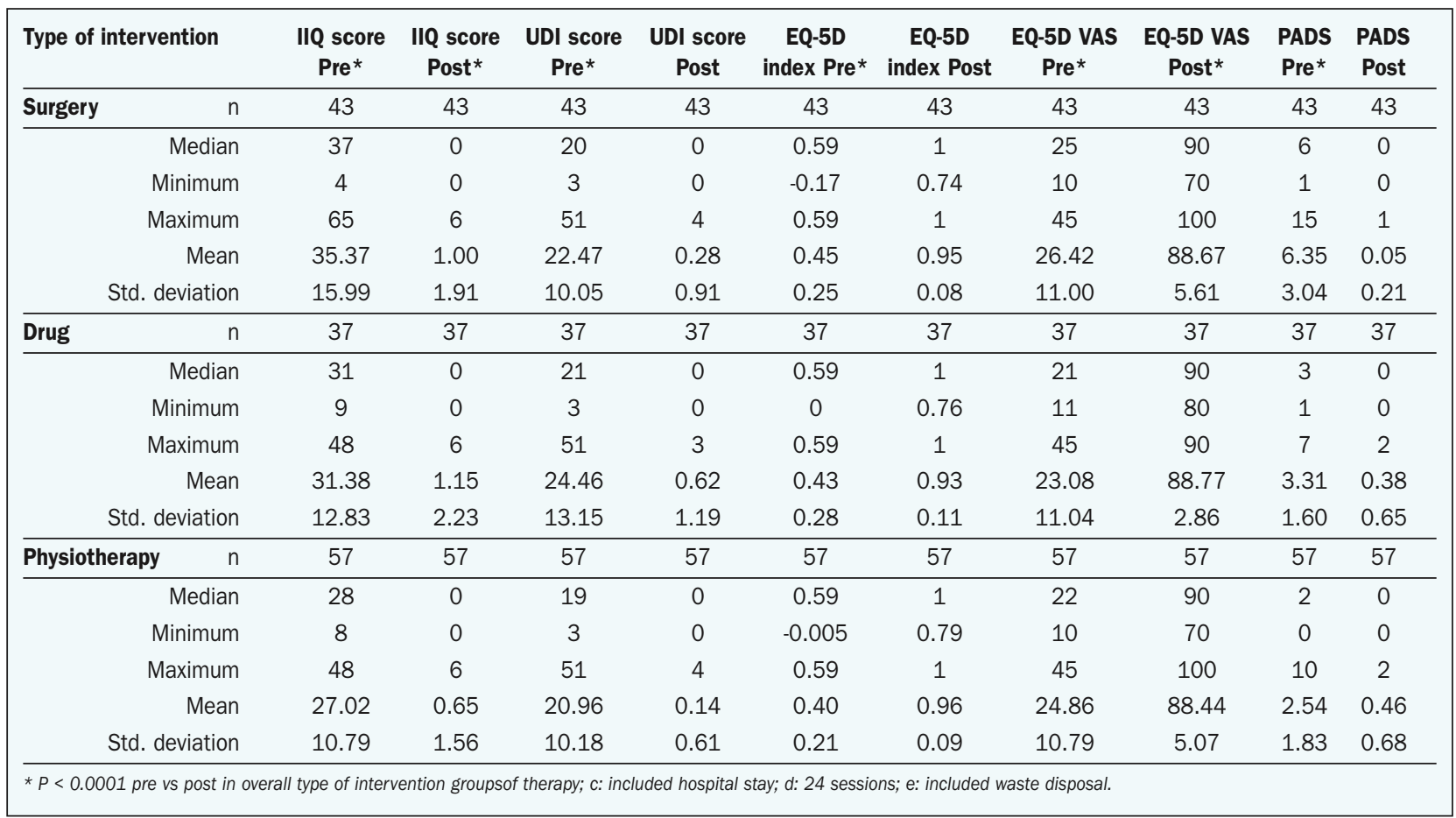

Table 3.

Cost-Utility Ratios (CUR) with $95 \% \mathrm{Cl}$ expressed in euros as the cost of each $Q A L Y$, according to both sections of the EQ-5D questionnaire and treatment.

\begin{tabular}{lccc|}
\hline Cost-Utility Ratio & Surgery & Drug & Physiotherapy \\
\hline EQ-5D Index & $702(582 ; 851)$ & $1453(1026 ; 2438)$ & $35(30 ; 42)$ \\
\hline EQ-5D VAS & $560(486 ; 651)$ & $1149(875 ; 1674)$ & $28(25 ; 32)$ \\
\hline
\end{tabular}

Table 4.

Change of the CUR (€) when pad use is factored in

\begin{tabular}{|lccc|}
\hline Cost-Utility Ratio & Surgery & Drug & Physiotherapy \\
\hline EQ-5D Index & $-735(-609 ;-890)$ & $995(703 ; 1701)$ & $-610(-529 ;-732)$ \\
\hline EQ-5D VAS & $-586(-509 ;-681)$ & $787(599 ; 1147)$ & $-494(-444 ;-563)$ \\
\hline
\end{tabular}

Table 5.

CUR including the cost of treating skin and urinary tract infections before and after treatment (estimated from published reports) (9).

\begin{tabular}{|lccc|}
\hline Cost-Utility Ratio & Surgery & Drug & Physiotherapy \\
\hline EQ-5D Index & $-1677(-1390 ;-2031)$ & $87(62 ; 149)$ & $-1519(-1317 ;-1822)$ \\
\hline EQ-5D VAS & $-1337(-1161 ;-1553)$ & $69(52 ; 100)$ & $-1229(-1104 ;-1402)$ \\
\hline
\end{tabular}

Since there are no Italian threshold values per QALY,we referred our findings to what the UK NICE proposed. According to NICE, costs are commonly classified as acceptable up to $€ 30000$ per QALY, as possibly acceptable between €30000$€ 45000$ per QALY and rejected over $€ 45000$ (11)

\section{Discussion}

We found that in an Italian regional setting appropriate diagnosis and treatment of incontinence may lower National Health Service costs and improve the benefits of treatment and quality of life. All interventions for incontinence showed a cost per QALY far below the acceptable cut-off according to NICE (10).

Over the past 25 years, health and medical services have become a major part of our economy. Applying economics to medical practice does not necessarily mean that less can or should be spent. Instead, the underlying belief is that resources should be allocated to treatments that maximize

Surgery and physiotherapy were associated with strong savings for each QALY and drug treatment was linked to a very low cost per QALY. According to both the EQ-5D Index and the EQ-5D VAS, the cost utility ratio was estimated at a saving of more than $€ 1200$ per QALY for surgery and physiotherapy. Drug therapy cost under $€ 100$ per QALY. social welfare (4). Cost-benefit analyses inform decision makers about how to allocate resources to maximize societal well-being within a limited budget

There are different typologies or frameworks for categorizing costs in health care e.g. direct, indirect and intangible costs. Direct costs for incontinence include diagnosis, treatment, routine care, (including cost of absorbent 
pads which, is one of the largest items), incontinencerelated consequences such as fractures from falls, nursing home admissions and UTI (12). However the causal link between IU and its consequences is less clear. Indirect costs are the value of lost productivity or lost employment due to morbidity (lost productivity and fewer hours of productive work). Intangible costs are the monetary value of pain and suffering. The present study included only direct costs because they are easier to assess. Costs of incontinence-related consequences were derived from a Spanish study in 2011 (10). Costs can also be categorized by type or by perspective (i.e who bears the cost: provider, society, patient etc.). Cost/utility, cost benefit and cost effectiveness analysis use the perspective category. In a cost of illness analysis (COI) analysis no attempt is made to measure the "value" of treatment while Cost-utility and Cost -benefit analysis address this particular issue. Cost effectiveness analysis (CEA) refers to the broad class of calculations where the effectiveness measure is a general health outcome. In studies on incontinence CEA focused on strategies for nursing home management (13-14), compared surgical techniques for stress incontinence (15-16), assessed pharmacological therapy of urgency incontinence and overactive bladder (17). Two studies using the perspective of the health care system $(16,18)$ provided evidence that trans-vaginal tape (TVT) is better than colposuspension although it remains unclear if the results would have been different if lifetime costs and benefits had been assessed and if a societal perspective had been included. There are many limitations to CEA and there has been widespread convergence on the use of QALYs as the preferred health outcome in cost-effectiveness analysis. Since the CUA is the gold standard in medicine (4) it was used in the present study. It refers to CEA using QALYs as the outcome measure: QALYs denote the relationship between the value of a given health state and the length of time a person lives in that health state. The value of a given health state is measured in "utilities" which represent preferences for a given health state.

The present study demonstrated that mid-urethral slings provided considerable savings ranging from 1337 to $1677 €$ per QALY, in patients with moderate-severe stress urinary incontinence as did physiotherapy in patients with mild stress urinary incontinence, which saved from 1229 to $1519 €$. These data confirm that appropriate diagnosis and treatment of incontinence lower National Health Service costs and clearly improve the benefits of treatment and quality of life.

A small but growing area of research has found that urinary incontinence and overactive bladder are associated with important and costly consequences (3) that can have a large impact on costs, morbidity, quality of life and mortality. Arlandis-Guzman $S$ et al. compared the economic value of overactive bladder (OAB) treatment with fesoterodine and extended release tolterodine and solifenacin from the social perspective (9). Their results were not comparable with ours because our study did not compare treatments as our aim was to evaluate the cost-utility of each treatment by examining costs and quality-adjusted life years. However, even though our drug treated group was small in number, the present study demonstrated that anti-cholinergic therapy with Solifenacin was linked to a much lower CUR than 30,000€ which the NICE adopted as the cost-useful threshold per QALY gained (11). Although the cost per QALY appears highly reasonable (less than $100 €$ ), reasons other than cost have to be taken into account before recommending this regimen to patients. Furthermore intermittent treatment may be suitable for some cases but needs in depth investigation to assess costs. Finally, variations in the cost of drugs from country to country becomes a pressing problem when the National Health Service does not fund the medication.

The major strength of the present study is that clinical research data derived mainly from GP referrals to the National Health Service Urology Unit in Perugia General Hospital. The entire cohort constitutes a representative sample from National Health Service records in a small region with no private medicine, meaning that all patients could be traced. Even though it might be objected that our evaluation is restricted to one Italian region and that costs as calculated in this paper would be completely different for each item (pads, surgery) in another healthcare system, we are of the view that Umbria provides a good example of a National Health Service model, which could be translated to other areas.

Although GP referrals should have ensured matched groups, groups were not in fact matched as allocation to a treatment option depended upon the type and severity of incontinence. Surgery, for example, was reserved for the worst cases. In the patients who underwent surgery we had no surgical complications and were unable to assess the costs of possible re-treatments due to the relatively short follow-up (1 year). However, data on midurethral sling outcomes confirm durability $(19,20)$ and low complication rates (21).

Other limitations of the present study are lack of a "usual care" arm in which either effectiveness or costs are modeled for comparison, the absence of perspective/ model, secondary effects, and inability to quantify the prevalence, and costs of treating, skin and urinary tract infections in our patients. In fact we had to resort to published reports for these figures. We were also unable to quantify the number, and treatment costs of episodes of depression. Therefore these three groups differed in incontinence severity and life expectancy. The costs of other pathologies and incontinence-related personal hygiene are unknown but probably similar in all three groups, though conceivably higher in patients who underwent surgery as they were affected by the most severe incontinence. Despite these differences drug therapy emerged as costing more than surgery and physiotherapy. Future investigations will have to attempt to create more homogeneous groups on age and life expectancy grounds. For ethical reasons severity of incontinence can never be uniformly distributed across groups.

Finally, in a certain percentage of patients incontinence is so severe that no treatment has any chance of success. The natural history of UI is not well understood but if it worsens over time, then rather than waiting until it becomes severe before starting therapy. early stage e.g. mild to moderate IU diagnosis and treatment would reduce National Health Service costs. 


\section{Conclusions}

This study shows that appropriate diagnosis and treatment of a patient with incontinence lowers National Health Service costs and clearly improves the benefits of treatment and quality of life.

\section{REFERENCES}

1. Hunskaar S, Burgio K, Diokno A, et al. Epidemiology and natural history of urinary incontinence in women. Urology. 2003; 62:Suppl 1:S16-23.

2. Thom $D$. Variation in estimates of urinary incontinence prevalence in the community: effects of differences in definition, population characteristics and study type. J Am Geriatr Soc. 1998; 46:473-80 .

3. Foxman B. Epidemiology of urinary tract infections: incidence, morbidity, and economic costs. Dis Mon. 2003; 49:53-70.

4. The-Wei Hu, TH Wagner, G Hawthorne, et al. Economics of incontinence in Incontinence Abrams P, Cardozo L, Kohury $S$ and Wein A (eds), Health Publication Ltd, Plymouth (UK) 2002; 14:965.

5. Gold M, Siegel JE, Russell L, Weinestein MC. (eds). Cost-effectiveness in health and medicine. Oxford University Press, Oxford. 1996.

6. Ingelman Sunderberg A, Ulmsten U. Surgical treatment of female urinary stress incontinence. Contrib Gynecol Obstet. 1983; 10:51-69.

7. Campbell MK, Torgenson DJ. Bootstrapping: Estimation confidence intervals for cost-effectiveness ratios. QJ Med. 1999; 92:177-82.

8. EuroQolGroup: EuroQol-A new facility for the measurement of health related quality of life. Health Policy. 1990; 16:199-208.

9. Dolan P. Modeling valutations for EuroQol health states. Med Care. 1997; 35:1095-108.

10. Arlandis-Guzman, et al. Cost-effectiveness analysis of antimuscarinics in the treatment of patients with overactive bladder in Spain: a decision-tre model. BMC Urology. 2011; 11:9.

11. National Institute for Health and Clinical Excellence (2012). Guide to the methods of technology appraisal. http://www.nice.org.uk/medial CB1/43/GuideToMethodsOfTechnologyAppraisal2012
12. Darkow T, Fontes CL, Williamson TE. Costs associated with the management of overactive bladder and related comorbidities. Pharmacotherapy. 2005; 25:511-9.

13. Schnelle JF, Keeler E, Simmons D, Ouslander, et al. A cost and value analysis of two interventions with incontinence nursing home residents. J Am Geriatr Soc. 1995; 43:1112-7.

14. Hu TW, Kaltreider DL, Igou LC, Rohner TJ. Cost effectiveness of training incontinent elderly in nursing homes: a randomized clinical trial. Health Serv Res. 1990; 25:455-77.

15. Ramsey SD, Wagner TH, Bavendam TG. Estimating costs of treating stress urinary incontinence in elderly women according to the AHCPR clinical practice guidelines. Am J Man Care. 1996; 2:147-54.

16. Manca A, Sculpher MJ, Ward K, Hilton P. A cost-utility analysis of tension-free vaginal tape versus colposuspension for primary urodynamic stress incontinence. BJOG. 2003; 110:255-62.

17. O’Brien BJ, Goeree R, Bernard L, , et al. Cost-effectiveness of tolterodine for patients with urge incontinence who discontinue initial therapy with oxybutinin: a Canadian perspective. Clin Ther. 2001; 23:2038-49.

18. Quievy A, Couturier F, Prudohn C, et al. Economic comparison of 2 surgical techniques for the treatment of stress urinary incontinence in women: Burch's technique versus the TVT technique. Prog Urol. 11: 347-53.

19. Albo ME, Litman HJ, Richter E, et al. for the Urinary Incontinence Treatment Network. Treatment Success of Retropubic and Transobturator Mid Urethral Slings at 24 Months. J Urol. 2012; 188:2281-7.

20. Porena M, Costantini E, Frea B, et al. Tension-free vaginal tape versus Transobturator tape as surgery for stress urinary incontinence: results of a multicentre randomised trial. Eur Urol. 2007; 52:1481-1490.

21. Novara G, Artibani W, Barber MD, et al. Updated systematic review and meta-analysis of the comparative data on colposuspensions, pubovaginal slings, and midurethral tapes in the surgical treatment of female stress urinary incontinence. Eur Urol. 2010; 58:218-38.

\author{
Correspondence \\ Elisabetta Costantini, MD \\ Massimo Lazzeri, MD \\ Alessandro Zucchi, MD (Corresponding Author) \\ azucchi@unipg.it \\ Emanuele Scarponi, MD \\ Massimo Porena, MD \\ Department of Medical-Surgical Specialties and Public Health \\ Urology and Andrology Section, Ospedale S. Maria della Misericordia \\ Loc. S. Andrea delle Fratte - 06100 Perugia, Italy \\ Vittorio Bini, MD \\ Department of Internal Medicine, University of Perugia, Perugia, Italy
}

